\title{
ARTICLE
}

\section{Wnt7b can replace Ihh to induce hypertrophic cartilage vascularization but not osteoblast differentiation during endochondral bone development}

\author{
Kyu Sang Joeng ${ }^{1,2,3}$ and Fanxin Long ${ }^{1,2,4,5}$
}

Indian hedgehog (Ihh) is an essential signal that regulates endochondral bone development. We have previously shown that $W n t 7 b$ promotes osteoblast differentiation during mouse embryogenesis, and that its expression in the perichondrium is dependent on Ihh signaling. To test the hypothesis that Wnt $7 \mathrm{~b}$ may mediate some aspects of Ihh function during endochondral bone development, we activated Wnt7b expression from the $\mathrm{R} 26-\mathrm{Wnt} 7 \mathrm{~b}$ allele with $\mathrm{Col}-\mathrm{Cre}$ in the $\mathrm{Ihh^{-/- }}$ mouse. Artificial expression of $\mathrm{Wnt} 7 \mathrm{~b}$ rescued vascularization of the hypertrophic cartilage in the $\mathrm{I} h \mathrm{~h}^{-/-}$mouse, but failed to restore orthotopic osteoblast differentiation in the perichondrium. Similarly, Wnt7b did not recover Ihh-dependent perichondral bone formation in the Ihh ${ }^{-/-}$; $\mathrm{Gli3}^{-/-}$embryo. Interestingly, Wnt7b induced bone formation at the diaphyseal region of long bones in the absence of Ihh, possibly due to increased vascularization in the area. Thus, Ihh-dependent expression of Wnt $7 \mathrm{~b}$ in the perichondrium may contribute to vascularization of the hypertrophic cartilage during endochondral bone development.

Bone Research (2014) 2, 14004; doi:10.1038/boneres.2014.4; published online 27 May 2014

\section{INTRODUCTION}

Indian hedgehog (Ihh) signaling is critical for endochondral bone development. Ihh is expressed by prehypertrophic and early hypertrophic chondrocytes, signaling to chondrocytes and perichondral cells. ${ }^{1-2}$ The studies of Ihh knockout mice have demonstrated the importance of Inh in chondrocyte proliferation and maturation, osteoblast differentiation and cartilage angiogenesis. ${ }^{2}$ Other genetic studies with smoothened (Smo), an essential transducer of hedgehog signaling, showed that direct Ihh input in chondrocytes and osteoprogenitors is required for chondrocyte proliferation and osteoblast differentiation, respectively. ${ }^{3-4}$ However, direct $\mathrm{Hh}$ signaling in endothelial cells is not necessary for vascularization of the hypertrophic cartilage, indicating that Ihh controls cartilage angiogenesis likely through a secondary signal. ${ }^{4}$

Wnt proteins are a family of glycoproteins playing critical roles in many aspects of animal development. During embryonic skeletal development, $\mathrm{Wnt} / \beta$-catenin signaling is required for osteoblast differentiation, as demonstrated by genetic studies of both $\beta$-catenin and the Wnt coreceptors Lrp5/6..$^{5-9}$ During endochondral bone development, osteoblast differentiation first occurs within the perichondrium surrounding the prehypertrophic and hypertrophic cartilage in response to Ihh. We term this process Ihh-dependent orthotopic perichondral ossification. Although Wnt/ $\beta$-catenin signaling has been shown to function downstream of Ihh during orthotopic perichondral ossification in the long bones, ${ }^{7-8}$ it is not known whether Wnt signaling can replace Ihh in this process. We have previously shown that Wnt7b is not only expressed in the domain of orthotopic perichondral ossification, but also required to ensure the timely initiation of the process. ${ }^{7}$ Interestingly, Wnt7b has also been shown by others to play important roles in proper vascularization of the central nervous system. ${ }^{10}$ A potential role for Wnt signaling in skeletal vascularization has not been examined.

\footnotetext{
'Department of Medicine, Washington University School of Medicine, St Lovis, MO, USA; ${ }^{2}$ Division of Biology and Biomedical Sciences, Washington University in St. Louis, St Lovis, MO, USA; ${ }^{3}$ Current address: Department of Molecular and Human Genetics, Baylor College of Medicine, Houston, TX, USA; ${ }^{4}$ Department of Orthopaedic Surgery, Washington University School of Medicine, St Louis, MO, USA and ${ }^{5}$ Department of Developmental Biology, Washington University School of Medicine, St Louis, MO, USA

Correspondence: F Long (longf@wudosis.wustl.edu)

Received: 27 December 2013; Revised: 10 January 2014; Accepted: 13 January 2014
} 
Here we test the hypothesis that Wnt7b may mediate Ihh function in inducing orthotopic perichondral ossification and hypertrophic cartilage vascularization. To this end, we artificially expressed Wnt7b in the endochondral skeleton of $/ h^{-1-}$ embryos. We provide evidence that Wnt7b is sufficient to induce vascularization of the hypetrophic cartilage in the absence of Ihh.

\section{MATERIALS AND METHODS}

Mouse strains

$\mathrm{Ihh}^{+/-}$, Gli3 ${ }^{+/-}$, Col2-Cre3 and R26-Wnt7b mouse strains were previously described..$^{2-3,11-12}$ All animal studies were approved by Washington University Animal Studies Committee.

\section{Analyses of mouse embryos}

For whole mount skeletal staining, we modified the McLeod method. ${ }^{13}$ Briefly, mouse embryos were isolated at E18.5. After removing skin, the embryos were fixed with 95\% ethanol overnight and incubated in acetone overnight. Next, embryos were stained with staining solution (0.03\% Alcian blue, $0.005 \%$ Alizarin Red S type, $10 \%$ glacial acetic acid, and 70\% Ethanol) for 2-3 days at room temperature. Stained embryos were incubated with $95 \%$ ethanol $24 \mathrm{~h}$, and then, cleared with $1 \% \mathrm{KOH}$. Finally, clear skeleton were stored in glycerol solution (8 part glycerol: 2 part $1 \% \mathrm{KOH}$ ). For histological and molecular analyses, embryonic limbs were harvested in cold PBS and fixed in $10 \%$ buffered formalin overnight at room temperature. Subsequently, the fixed limbs were decalcified with $14 \%$ EDTA/PBS ( $\mathrm{pH} 7.4$ ) for 3 days. Finally, the limbs were processed and embedded in paraffin for sectioning $(6 \mu \mathrm{m}$ thickness). Limb sections were stained with hematoxylin and eosine (H\&E) for basic histological analysis. Alcian blue and picro-sirius red ${ }^{14}$ staining were performed to distinguish cartilage and bone matrix on sections. Radioactive in situ hybridization using ${ }^{35}$ S-labeled riboprobes was performed as previously described. ${ }^{3-4,7,15}$

\section{RESULTS}

Wnt7b expression in perichondrium requires Ihh

Previously, in E14.5 mouse embryos, we have shown that Wnt7b is expressed by perichondral cells flanking the prehypertrophic and early hypertrophic cartilage, and that the expression is critically dependent on Ihh signaling. To corroborate this finding, we performed in situ hybridization on long bone sections from mouse embryos of later stages. At E15.5, Wnt7b was normally expressed most robustly in the perichondrium flanking the prehypertrophic and early hypertrophic chondrocytes, but also at a lower level in the perichondrium/periosteum flanking the emerging marrow cavity (Figure lal and 1a2). Interestingly, at E18.5, Wnt7b expression was more restricted to the perichondrium surrounding the prehypertrophic and early hypertrophic chondrocytes, with no obvious expression in the diaphyseal periosteum (Figure $1 \mathrm{bl}$ and $1 \mathrm{~b} 2$ ). In contrast, Wnt7b was not detected in Ihh ${ }^{-1-}$ embryos at either stage (Figure $1 \mathrm{cl}, 1 \mathrm{c} 2,1 \mathrm{~d} 1$ and $1 \mathrm{~d} 2$ ). Thus, Wnt7b is expressed in the osteogenic perichondrium in an Ihhdependent manner.

\section{Overexpression of Wnt7b increases embryonic bone} formation

To study the role of Wnt7b on bone formation, we took advantage of the R26-Wnt7b mouse strain that can overexpress Wnt7b in a Cre-dependent manner. ${ }^{12}$ Specifically, we generated Col2-Cre3;R26 $6^{\text {Wht7b/+ }}$ mice (termed C2Wnt7b mice) by using the Col2-Cre3 transgenic line that targets both osteoblasts and chondrocytes. ${ }^{3}$ Wholemount skeletal staining at E18.5 indicated that the long bones in $\mathrm{C} 2 \mathrm{WnT7b}$ embryos were slightly shorter but noticeably thicker than those in the normal littermate with the genotype of $R 26^{W n t 7 b /+}$ (Figure $2 a$ and b). Histological analyses showed not only an increase in the thickness of the cortical bone, but also a presumptive marrow cavity filled with bone in the C2Wnt7b animal (Figure $2 c, 2 c 1,2 d$ and $2 \mathrm{~d} 1 \mathrm{l}$. On the other hand, the growth plate cartilage appeared to be relatively normal. Consistent with histology showing extra bone mass within the presumptive marrow cavity of $\mathrm{C} 2 \mathrm{Wnt7b}$ mice, in situ hybridization confirmed that the area contained an abnormally large number of osteoblasts expressing the well-known markers including Osterix (Osx), bone sialoprotein (Bsp), and osteocalcin (OC) (Figure 3). These results confirm that the R26Wnt7b mouse strain is functional and that Wnt7b stimulates bone formation in the mouse embryo.

Wnt7b induces hypertrophic cartilage vascularization in Inh ${ }^{-1-}$ embryos

To test whether Wnt7b mediates Ihh function during endochondral bone development, we produced Col2-Cre3; $\mathrm{Ihh}^{-1-}$; R26 $6^{\text {Wnt7b/+ }}$ (termed Wnt7b-rescue) embryos by

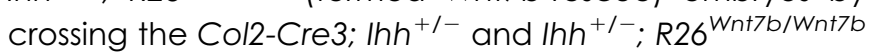
mice. The $1 \mathrm{hh}^{-1-} ; \mathrm{R} 26^{\mathrm{Wn} t 7 \mathrm{~b} /+}$ embryos, like the $\mathrm{Ih}^{-1-}$ embryos as previously reported, showed little vascularization of the hypertrophic cartilage at E18.5, even though the control littermate had developed a bone marrow cavity (Figure $4 \mathrm{al}, 4 \mathrm{a} 2,4 \mathrm{~b} 1$ and $4 \mathrm{~b} 2$ ). The long bones of the Wnt7b-rescue embryos appeared to be similar in gross morphology to those of $1 \mathrm{hh}^{-1-}$; $R 26^{\text {Wnt7b/+ }}$ embryos (Figure $4 \mathrm{bl}$ and $4 \mathrm{cl}$, and data not shown). However, they displayed clear vascularization in their hypertrophic cartilage, as evident by the presence of red blood cells, even though a marrow cavity was not formed (Figure $4 \mathrm{cl}$ and $4 c 2$ ). To gain further insight about the vascularization phenotype, we performed in situ hybridization with 


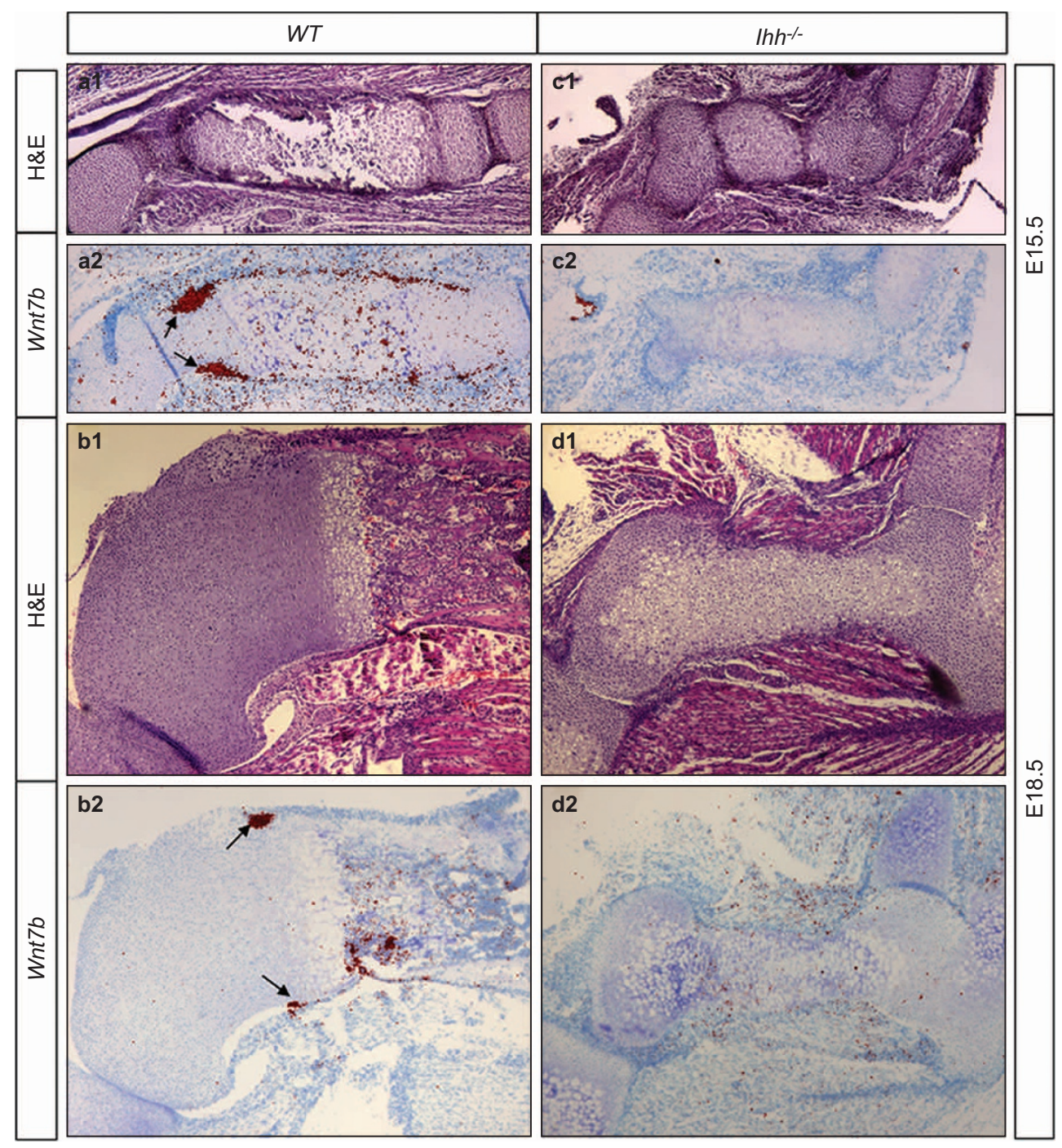

Figure 1. Expression of Wnt7b in developing long bones of mouse embryos. (a1, a2, b1, b2) H\&E staining (a1, b1) and Wnt7b in situ hybridization (a2, b2) on longitudinal sections of the humerus in WT embryos at E15.5 (a1, a2) and E18.5 (b1, b2). (c1, c2, d1, d2) H\&E staining (c1, d1) and Wnt7b in situ hybridization (c2, d2) on longitudinal sections of the humerus in $I h h^{-/-}$embryos at E15.5 (c1, c2) and E18.5 (d1, d2). In situ hybridization signal in red. Arrows denote expression in perichondrium flanking prehypertrophic and early hypertrophic cartilage. WT, wild-type.

molecular markers for hypertrophy and vascularization. Normally, Colloal is most robustly expressed by the early and intermediate hypertrophic chondrocytes, whereas Mmp 13 demarcates the terminal hypertrophic cells as well as osteoblast-lineage cells, and Mmp9 marks the leading edge of vascular invasion in the bone marrow cavity (Figure $4 \mathrm{~d} l-4 \mathrm{~d} 3$ ). In the $1 \mathrm{hh}^{-1-}$; $R 26^{\mathrm{Wnt7b} /+}$ embryo, the core of the cartilage element contained a hypertrophic domain expressing Colloal peripherally and Mmpl3 centrally in a concentric manner, but no $\mathrm{Mmp} 9$ expression was detected, confirming no vascularization of the hypertrophic cartilage (Figure 4el-4e3). In contrast, expression of Colloal and Mmpl3 in the hypertrophic cartilage of Wnt7b-rescue mice assumed a linear instead of concentric arrangement more similar to the normal pattern (Figure $4 \mathrm{f} 1$ and 4f2). More importantly, the Wnt7b-rescue cartilage expressed Mmp9 within the hypertrophic region, indicative of vascular invasion (Figure 4f3). Likely as a result of the vascularization, the Mmp13-positive domain was no longer contiguous in the central hypertrophic region in the Wnt7b-rescue embryo, in contrast to the $\mathrm{Ih} \mathrm{h}^{-1-}$; R26 $6^{\text {Wnt7b/+ }}$ mutant (Figure $4 \mathrm{e} 2$ and $4 \mathrm{f} 2$ ). Overall, forced expression of Wnt7b was sufficient to induce hypertrophic cartilage angiogenesis in the absence of Ihh.

Wnt7b expression does not rescue Ihh-dependent osteoblast differentiation in $\mathrm{hh}^{-1-}$ mutants

We next analyzed the potential rescue of osteoblast differentiation in Wnt7b-rescue embryos. Histology indicated no cortical bone (bone collar) or primary spongiosa in the Wnt7b-rescue embryo (Figure $4 \mathrm{cl}$ and $4 \mathrm{c} 2$ ). To gain more insight, we performed molecular analyses for osteoblast 


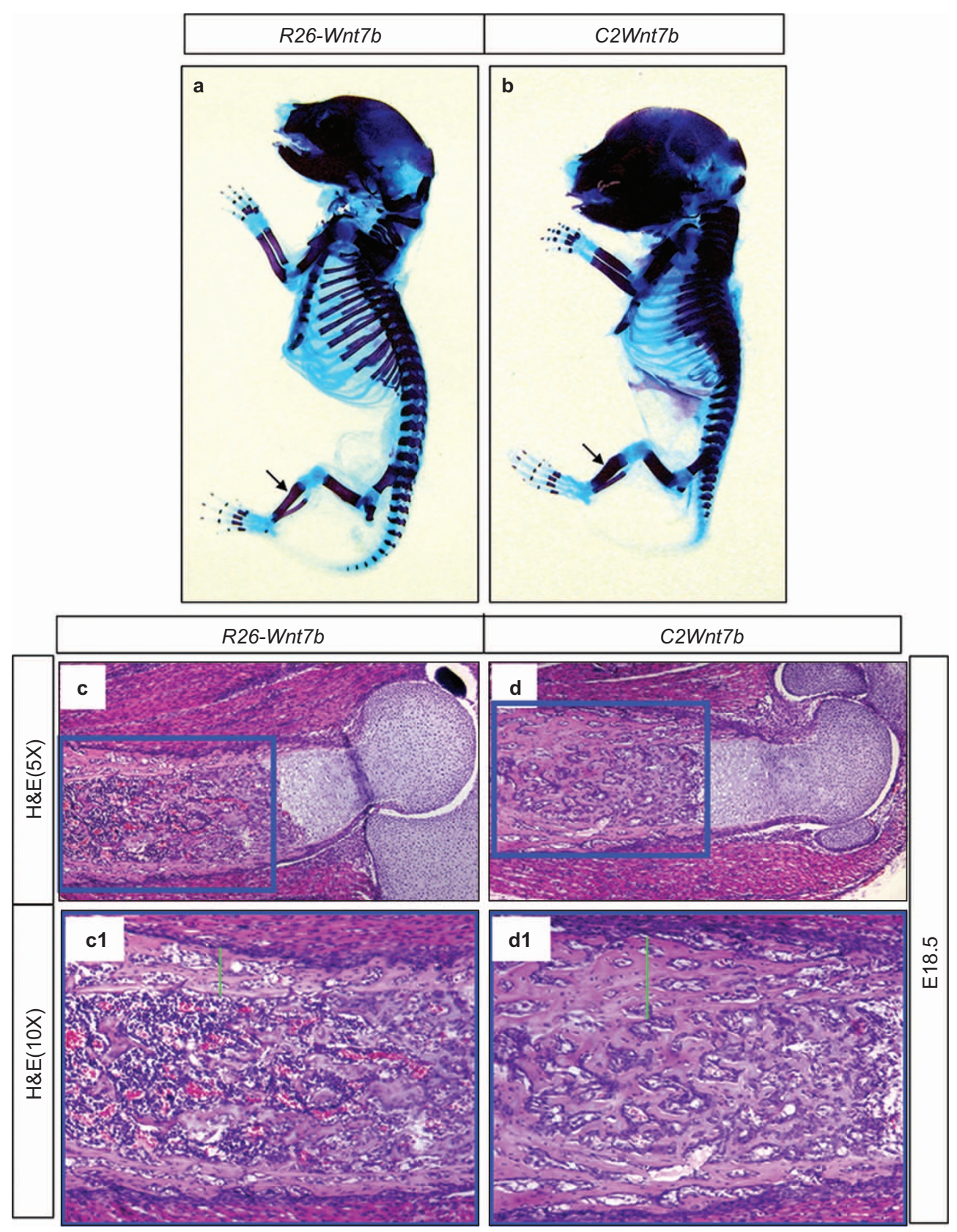

Figure 2. Wnt7b increases bone formation during endochondral bone development. (a, b) Whole-mount skeletal staining of E18.5 embryos from $R 26^{\text {Wnt } 7 b /+}$ (a) versus Col2-Cre3; R26 $6^{W n t 7 b /+}$ (b) embryos. Arrows point to tibia that show marked size difference. (c, d) H\&E staining of longitudinal sections through the distal half of humerus. (c1, d1) Higher magnification of boxed regions shown in $\mathbf{c}$ and $\mathbf{d}$. Green line in c1, d1 denotes cortical thickness.

differentiation. In situ hybridization revealed normal expression patterns for Runx2, AP and Osx in E18.5 $\mathrm{lhh}^{+/-}$ embryos. These molecules were detected in the primary spongiosa, the diaphyseal periosteum (red arrow) as well as the perichondrium flanking the prehypertrophic and hypertrophic cartilage (blue arrow) (Figure 5al-5a3). The last domain represents the area wherein Ihh-dependent osteoblast differentiation (orthotopic perichondrial ossification) occurs during normal development. In contrast, no such expression was detected in the perichondrium of $I \mathrm{hh}^{-1-} ; \mathrm{R} 26^{\mathrm{Wnt} 7 \mathrm{~b} /+}$ long bones, even though the hypertrophic cartilage expressed Runx2 (Figure 5bl-5b3). Compared to $1 \mathrm{hh}^{-1-} ; \mathrm{R}_{2} 6^{\mathrm{Wnt} 7 \mathrm{~b} /+}$ embryos, the long bones of Wnt7b-rescue embryos also lacked the osteoblast markers in the perichondrium flanking the prehypertrophic and hypertrophic cartilage (blue arrow) (Figure $5 c 1-5 c 3$ ). Interestingly, however, Runx2, AP and Osx were detected in the diaphyseal perichondrium in the Wnt7b-rescue embryo, typically on one side of the long bone (red arrow), and also within the presumptive 


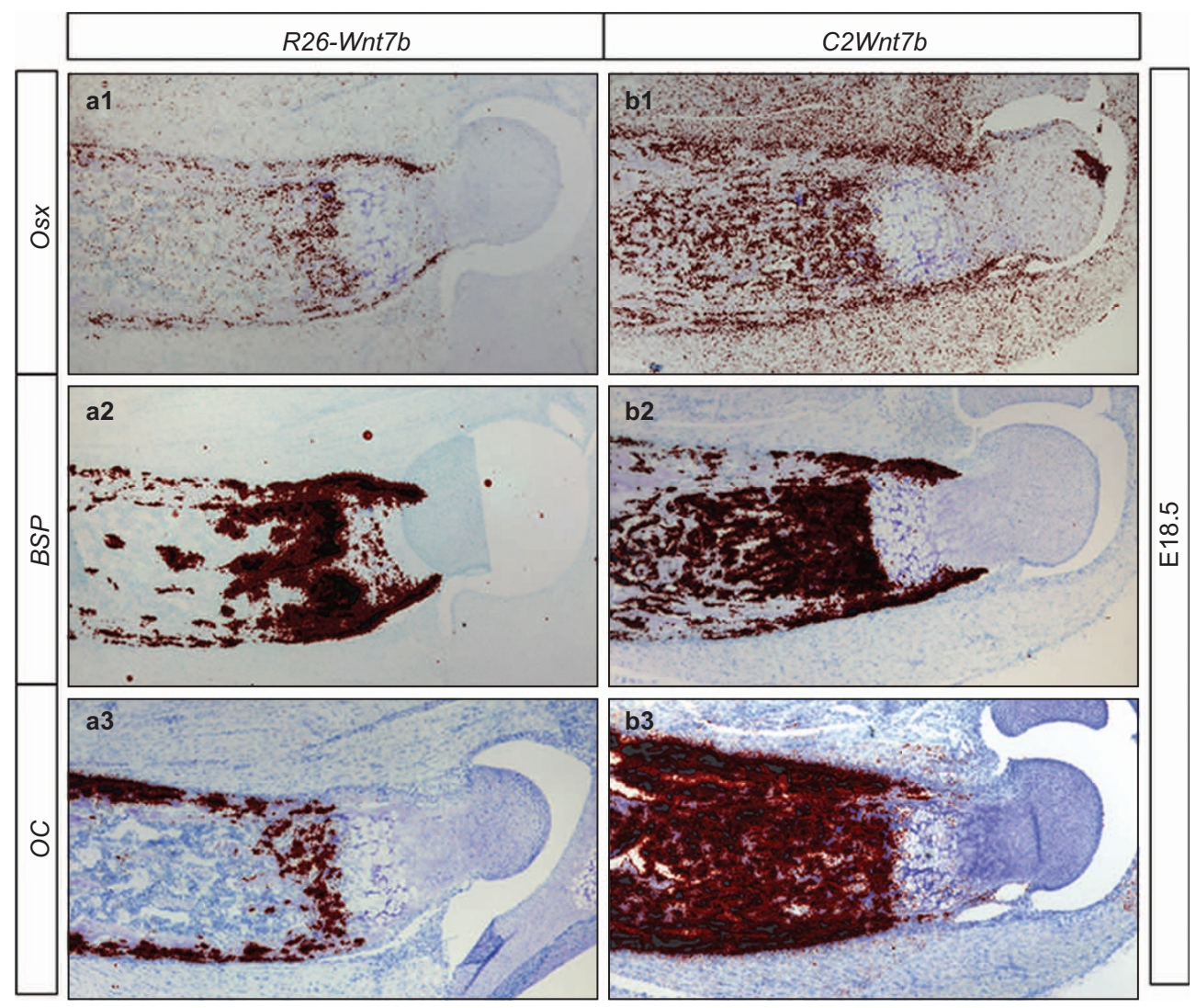

Figure 3. Molecular analyses of osteoblast differentiation at E18.5. In situ hybridization of osteoblast markers on sections of distal humeri from $R 26^{\text {Wnt7b/+ }}(\mathbf{a 1 - a 3})$ versus Col2-Cre3; R26 $6^{\text {Wnt7b/+ }}$ (b1-b3) littermate embryos at E18.5. In situ hybridization signals shown in red.

marrow region (asterisk) (Figure $5 c 1-5 c 3$ ). Because expression of the osteoblast markers in these areas coincided with vascularization, the induction of osteoblast differentiation may be secondary to the increased vascularization in response to Wnt7b. A formal proof for this hypothesis awaits further studies. In summary, Wnt7b is not sufficient to restore orthotopic perichondral ossification in the $I \mathrm{hh}^{-1-}$ embryo, but appears to induce osteoblast differentiation secondary to the enhanced vascularization.

Wnt7b expression does not rescue Ihh-dependent osteoblast differentiation in $\mathrm{hh}^{-1-} ; \mathrm{Gli3}^{-1-}$ embryos Wnt7b expression in $/ \mathrm{hh}^{-1-}$ mutant enhanced hypertrophic cartilage angiogenesis, but failed to rescue Ihhdependent osteoblast differentiation. This phenotype was remarkably similar to that of the $\Delta$ NGli2-rescue embryo. ${ }^{16}$ Because removal of $\mathrm{Gli} 3$ in the $\Delta$ NGli2-rescue embryo rescued lhh-dependent orthotopic perichondral ossification, we hypothesized that the process can be similarly restored by simultaneous removal of Gli3 and expression of Wnt7b. To test this hypothesis, we generated Col2-Cre3; Ihh ${ }^{-1--}$; $\mathrm{Gli3}^{-1-}$; R26 $6^{\text {Wnt7b/+ }}$ embryos (termed double-rescue embryo) by crossing Col2-Cre3; $\mathrm{Ih}^{+/-}$; $\mathrm{Gli3}^{+/-}$with Ihh ${ }^{+/-}$; $\mathrm{Gli3}^{+/-} ; \mathrm{R}^{2} 6^{\text {Wnt7b/Wnt7b }}$ mice.
We first performed histological analyses on the long bones at E18.5. The length and organization of the growth plate was largely restored in the double-rescue embryo (Figure $6 a 1, b 1, a 2$ and b2). This was consistent with our previous finding that removal of Gli3 restored growth plate morphology in the $\mathrm{Ihh}^{-1-}$ embryo. ${ }^{15}$ Interestingly, the double-rescue embryo formed a bone collar detectable by either H\&E or sirus red staining (Figure $6 \mathrm{bl}$ and $6 \mathrm{~b} 2$ ). Careful examination revealed that bone was formed at the diaphyseal region surrounding the presumptive bone marrow cavity, but not in the perichondrium surrounding the hypertrophic zone where ossification normally occurs (Figure 6b4). The presumptive marrow cavity also contained bone trabeculae in the double-rescue embryo (Figure 6b3). The diaphyseal bone formation in the double-rescue mouse was similar to that observed in $\mathrm{hh}^{-1-}$; Gli3 $^{-1-}$ embryos, but appeared to be more robust ${ }^{15}$ (data not shown). However, because our current mating strategy did not produce $/ \mathrm{hh}^{-1-} ; \mathrm{Gli3}^{-1-}$ littermate embryos for direct comparison, we cannot rule out the possibility that the apparent quantitative difference might be due to differences in genetic background.

To confirm the bone phenotype at the molecular level, we examined the expression of osteoblast markers 


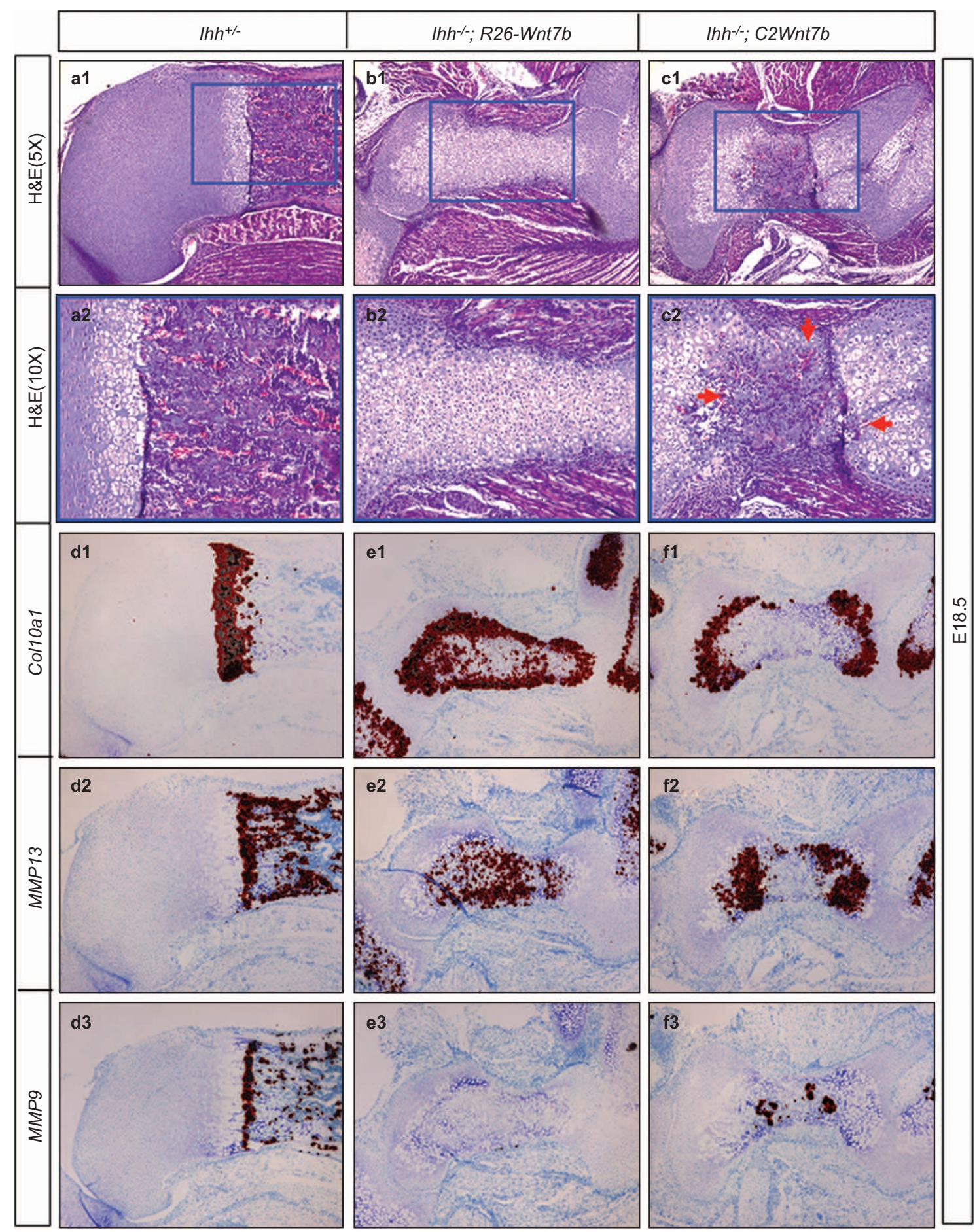

Figure 4. Analyses of hypertrophic cartilage vascularization in the humerus of E18.5 littermate embryos. (a1-c1) H\&E staining of humerus sections

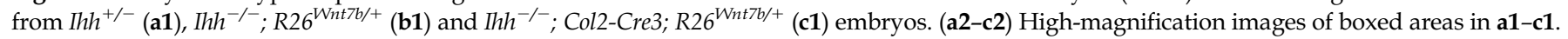
Red arrows point to red blood cells. (d1-d3, e1-e3, f1-f3) In situ hybridization on humerus sections. Hybridization signal shown in red.

including Runx2, Osx, Bsp and OC. These markers were normally expressed by all osteoblasts associated with the primary spongiosa, diaphyseal periosteum (red arrow) and the perichondrium flanking the prehypertrophic and hypertrophic cartilage (blue arrow) (Figure 7al-7a4).
However, in the double-rescue embryo, although the markers were expressed in the diaphyseal region including the periosteum (red arrow), they were not present at any significant level in the presumptive region for orthotopic perichondral ossification (blue arrow) (Figure 7b1-7b4). 


\begin{tabular}{|c|c|c|}
\hline$I h h^{+/}$ & $I h h^{-/} ; R 26-W n t 7 b$ & $I h h^{-/} ; C 2 W n t 7 b$ \\
\hline
\end{tabular}
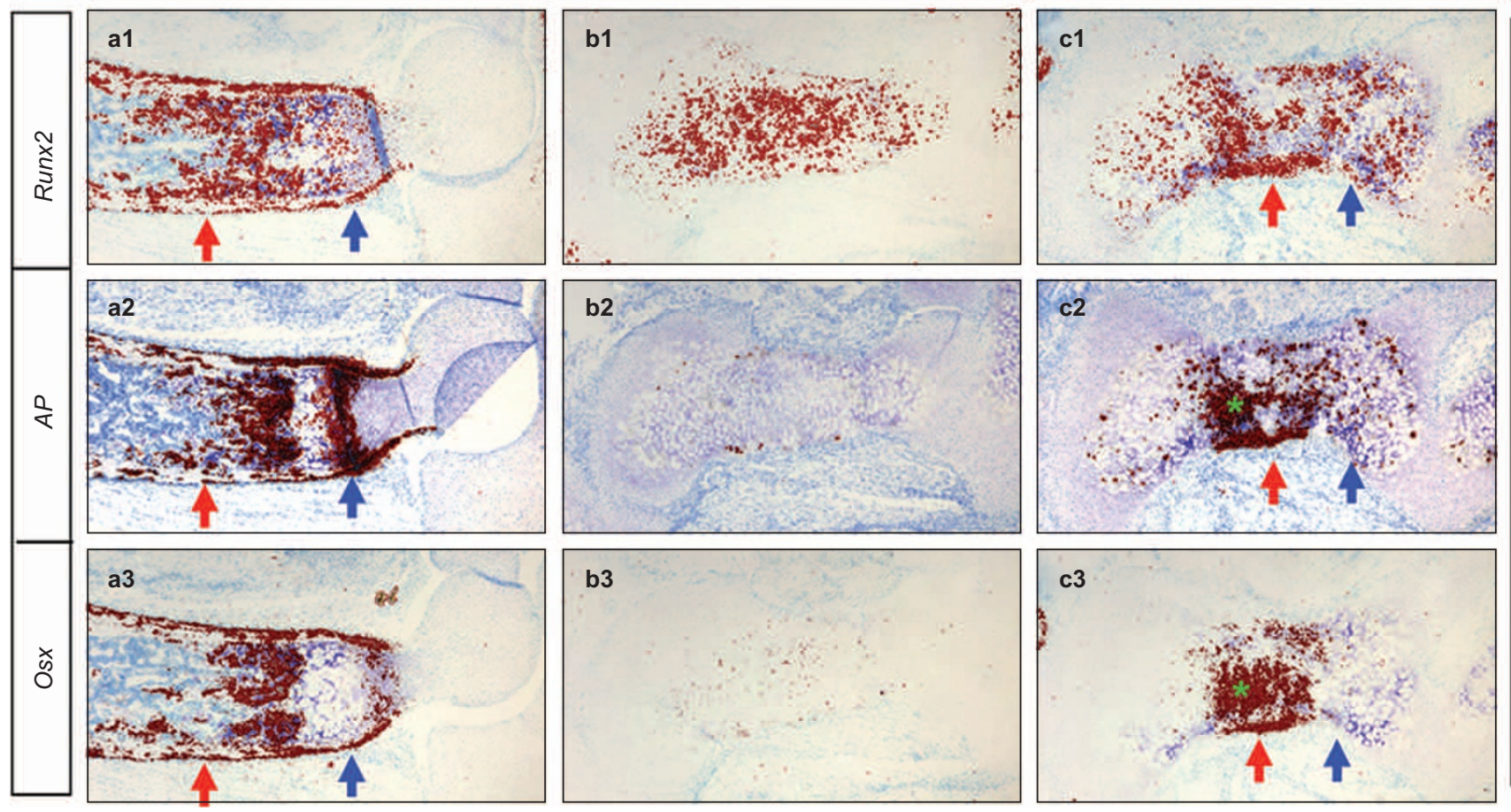

Figure 5. Molecular analyses for osteoblast differentiation in E18.5 littermate embryos. In situ hybridization of Runx2 (a1-a3), AP (b1-b3) and Osx (c1c3) on humerus sections. Distal end to the right. Blue arrows: perichondrium flanking hypertrophic chondrocytes; red arrows: diaphyseal perichondrium. Asterisk denotes signal in presumptive marrow cavity.

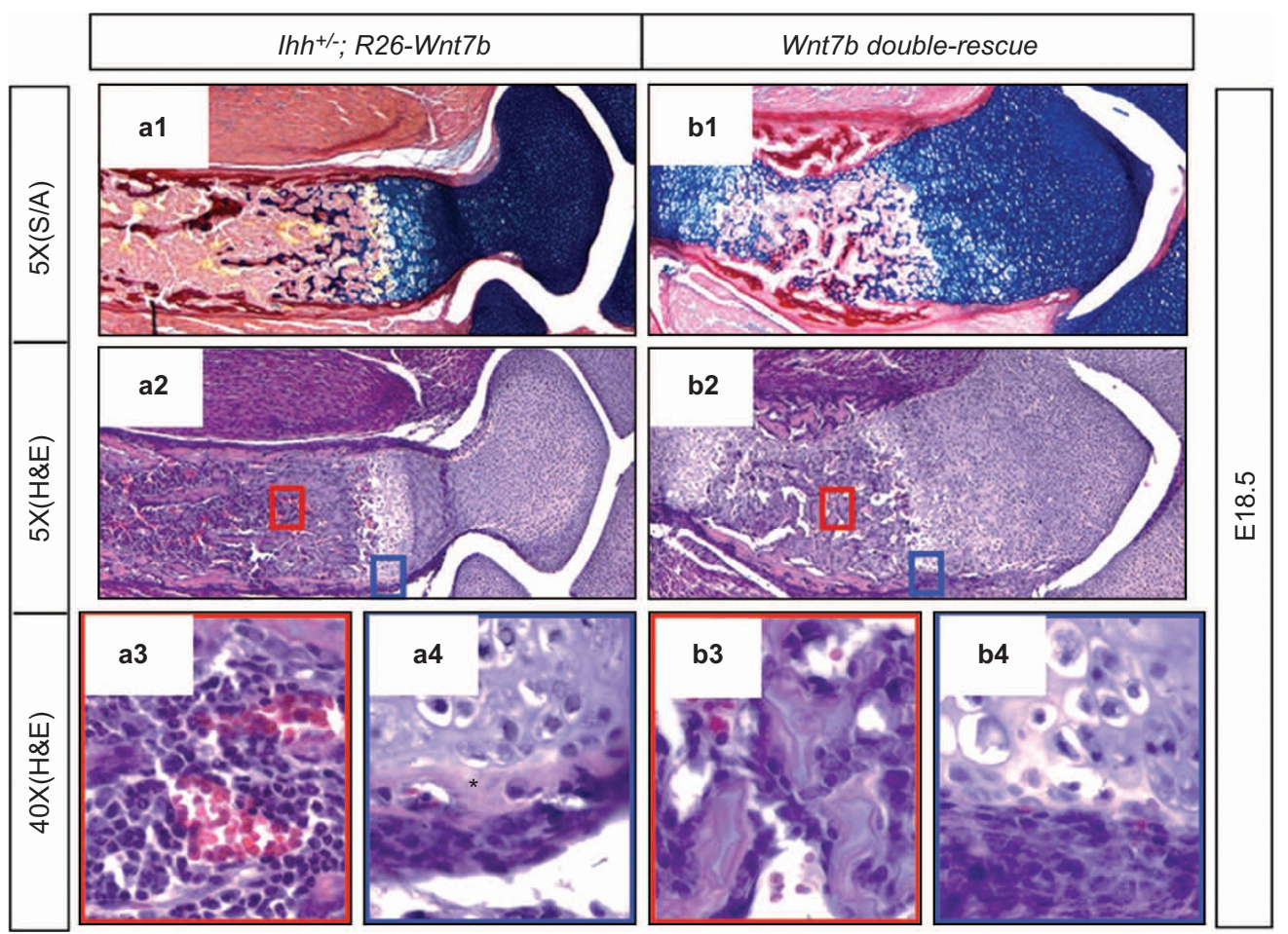

Figure 6. Morphological analyses of $I h h^{+/-} ; R 26^{\text {Wnt7b/+ }}$ versus Col2-Cre3; $I h h^{-/-} ; G l i 3^{-/-} ; R 26^{\text {Wnt7b/+ }}$ (Wnt7b double rescue) littermate embryos at E18.5. (a1, b1) Sirius Red and Alcian Blue staining of longitudinal sections through the humerus. Distal end to the right. (a2, b2) H\&E staining. (a3, a4, b3, b4) Higher magnification images of boxed areas in $\mathbf{a} 2$ and $\mathbf{b} \mathbf{2}$. 


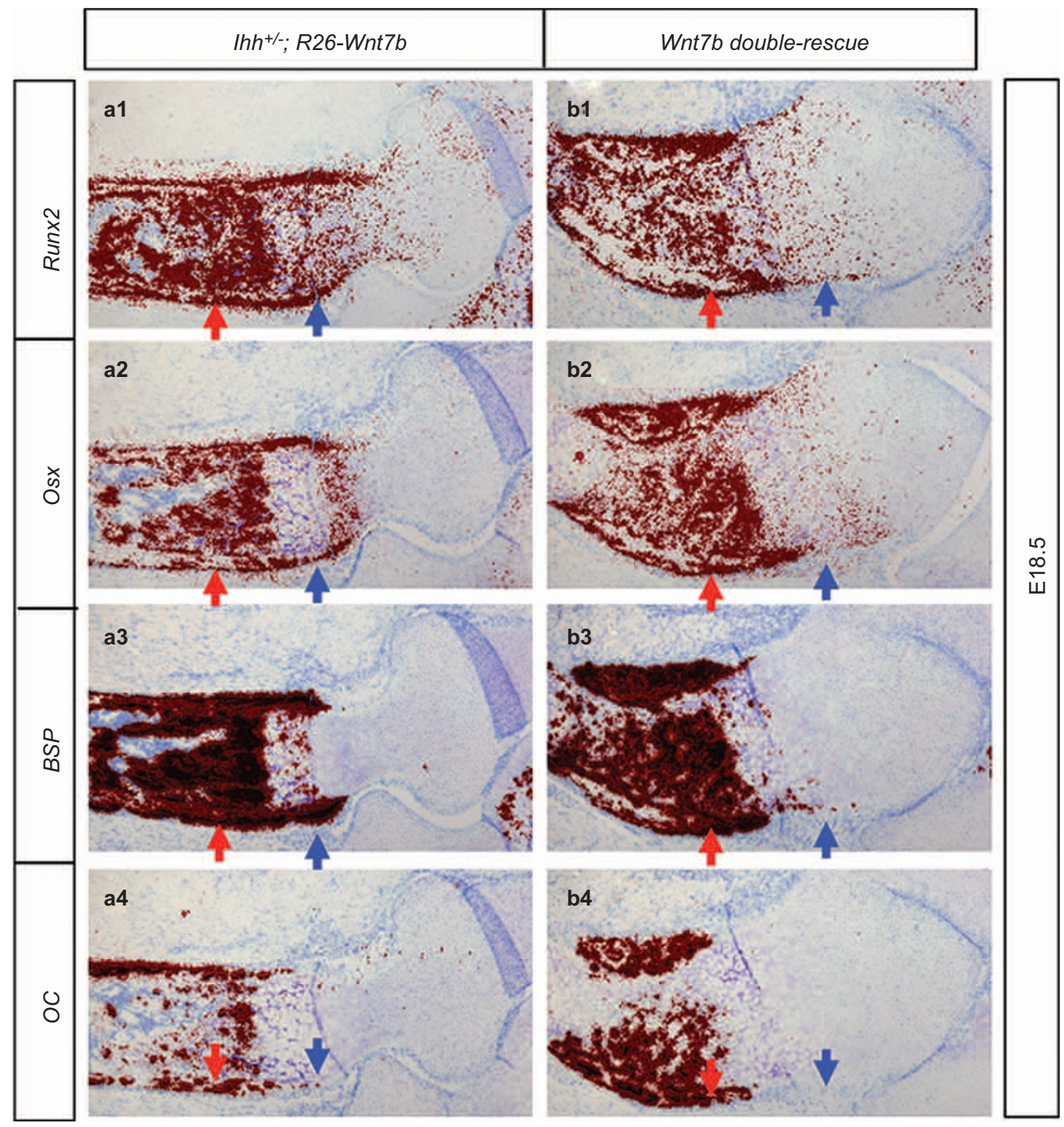

Figure 7. In situ hybridization for osteoblast markers in $I h h^{+/-} ; R 26^{\mathrm{Wn} t 7 b /+}$

(a1-a4) versus Col2-Cre3; $\mathrm{Ihh}^{-/-} ; \mathrm{Gli3}^{-/-} ; \mathrm{R}^{2} 6^{\mathrm{Wnt} 7 \mathrm{~b} /+}$ (Wnt7b double rescue) (b1-b4) littermate embryos at E18.5. Shown are humerus sections with distal end to the right. Blue arrow: perichondrium flanking hypertrophic chondrocytes; red arrow: perichondrium flanking diaphysis.

Thus, Wnt7b expression coupled with Gli3 deletion did not restore orthotopic perichondral ossification in the $\mathrm{Ih}^{-1-}$ embryo.

\section{DISCUSSION}

We have investigated the potential role of Wnt7b in mediating the multiple functions of Ihh signaling during endochondral bone development. By examining mouse embryos at multiple embryonic stages, we confirmed the Wnt7b is expressed by perichondrial cells undergoing Ihhdependent ossification and that the expression is dependent on Ihh. Through genetic experiments that forceexpressed Wnt7b, we found that Wnt7b was sufficient to induce hypertrophic cartilage vascularization that is otherwise absent in I $\mathrm{hh}^{-1-}$ embryos. On the other hand, Wnt7b forced-expression failed to activate perichondrial osteoblast differentiation at the orthotopic position when Inh was absent, even when the transcription repressor Gli3 was removed. These results provide additional insights about the mechanisms of Ihh function during endochondral skeletal development.

How thh regulates Wnt7b expression is not understood at present. We have noticed in the Wnt7b promoter potential Gli binding sites conforming to the consensus sequence TGG GTG GTC as previously defined by others, ${ }^{17-18}$ so it will be of interest to determine whether these sites mediate direct regulation of Wnt7b expression by Ihh. Alternatively, the control of Ihh over Wnt7b expression may be indirect.

It is noteworthy that Wnt7b expression caused osteoblast differentiation within the diaphyseal perichondrium in the absence of Ihh. Although at the histological level, 
bone tissue was not evident in the Wnt7b-rescue embryo, molecular analyses revealed upregulation of Runx2, AP and Osx within a small domain of the perichondrium at the diaphysis, as well as in the presumptive marrow cavity. Interestingly, expression of the osteoblast markers was invariably detected asymmetrically on one side of the long bone section, which exhibited more advanced vascularization (judged by histology) than the other side. A similar concurrence between diaphyseal ossification and vascularization was observed in $/ \mathrm{hh}^{-1-} ; \mathrm{Gli3}^{-1-}$ embryos as we have previously reported..$^{15}$ Indeed, when Wnt7b was force-expressed in the background of $\mathrm{hh}^{-1-} ; \mathrm{Gli3}^{-1-}$, diaphyseal bone formation was most prominent, as indicated by both histology and molecular analyses. These results support the hypothesis that vascularization was sufficient to activate the osteogenic program within diaphyseal perichondrium independent of Ihh. Future studies are necessary to elucidate the osteogenic signals associated with vascular invasion.

In contrast to the diaphyseal perichondrium, the perichondrium flanking the prehypertrophic and hypertrophic cartilage requires Ihh signaling to undergo osteoblast differentiation. We have previously shown that simultaneous activation of Gli2 and removal of Gli3 can replace Ihh to activate osteogenesis within this domain. ${ }^{15-16}$ Others reported that Glil also plays a stimulatory role in this process. ${ }^{19}$ Downstream of the Gli transcription factors, however, the critical effectors responsible for osteoblast differentiation are not known. We previously demonstrated that forced-expression of Runx2 failed to restore orthotopic perichondral ossification in $1 \mathrm{hh}^{-1-}$ embryos. ${ }^{20}$ Similarly, here we show that Wnt7b cannot replace Ihh in this process. Thus, induction of perichondral ossification by Ihh likely involves multiple downstream effectors, and a full understanding of the process requires further investigation.

In contrast to osteoblast differentiation, vascularization of the hypertrophic cartilage was induced by forcedexpression of Wnt7b in the absence of Ihh. The evidence for Wnt7b as an angiogenic signal previously came from studies of the central nervous system, but a similar role in other systems has not been demonstrated. ${ }^{10,21}$ The present study indicates that Wnt7b normally produced by the perichondrium may function as a relay signal for the chondrocyte-derived lihh to induce blood vessel invasion of the hypertrophic cartilage. It is not known at present whether a direct input of Wnt signaling in endothelial cells is required for cartilage angiogenesis. To answer that question would require manipulation of Wht signal reception or transduction directly in the endothelium.

\section{Conflict of Interest}

The authors declare no conflict of interest.

\section{Acknowledgements}

This work is supported by NIH grants R01 DK065789 and R01 AR060456 to FL.

\section{References}

1 Vortkamp A, Lee K, Lanske B et al. Regulation of rate of cartilage differentiation by Indian hedgehog and PTH-related protein. Science 1996; 273: 613-622.

2 St-Jacques B, Hammerschmidt M, McMahon AP. Indian hedgehog signaling regulates proliferation and differentiation of chondrocytes and is essential for bone formation. Genes Dev 1999; 13: 2072-2086.

3 Long F, Zhang XM, Karp S, Yang Y, McMahon AP. Genetic manipulation of hedgehog signaling in the endochondral skeleton reveals a direct role in the regulation of chondrocyte proliferation. Development 2001; 128: 5099-5108.

4 Long F, Chung UI, Ohba S et al. Thh signaling is directly required for the osteoblast lineage in the endochondral skeleton. Development 2004; 131: 1309-1318

5 Day TF, Guo X, Garrett-Beal L, Yang Y. Wnt/beta-catenin signaling in mesenchymal progenitors controls osteoblast and chondrocyte differentiation during vertebrate skeletogenesis. Dev Cell 2005; 8: 739 750.

6 Hill TP, Spater D, Taketo MM, Birchmeier W, Hartmann C. Canonical Wnt/beta-catenin signaling prevents osteoblasts from differentiating into chondrocytes. Dev Cell 2005; 8: 727-738.

$7 \mathrm{Hu} \mathrm{H}$, Hilton MJ, Tu X et al. Sequential roles of Hedgehog and Wnt signaling in osteoblast development. Development 2005; 132: 49-60.

8 Rodda SJ, McMahon AP. Distinct roles for Hedgehog and canonical Wnt signaling in specification, differentiation and maintenance of osteoblast progenitors. Development 2006; 133: 3231-3244.

9 Joeng KS, Schumacher CA, Zylstra-Diegel CR, Long F, Williams BO. Lrp5 and Lrp6 redundantly control skeletal development in the mouse embryo. Dev Biol 2011; 359: 222-229.

10 Stenman JM, Rajagopal J, Carroll TJ et al. Canonical Wnt signaling regulates organ-specific assembly and differentiation of CNS vasculature. Science 2008; 322: 1247-1250.

11 Maynard TM, Jain MD, Balmer CW, LaMantia AS. High-resolution mapping of the Gli3 mutation extra-toes reveals a $51.5-\mathrm{kb}$ deletion. Mamm Genome 2002; 13: 58-61.

12 Chen J, Tu X, Esen E et al. WNT7B promotes bone formation in part through mTORC1. PLoS Genet 2014; 10: e1004145.

13 McLeod MJ. Differential staining of cartilage and bone in whole mouse fetuses by alcian blue and alizarin red S. Teratology 1980; 22: 299-301.

14 Junqueira LC, Bignolas G, Brentani RR. Picrosirius staining plus polarization microscopy, a specific method for collagen detection in tissue sections. Histochem J 1979; 11: 447-455.

15 Hilton MJ, Tu X, Cook J, Hu H, Long F. Ihh controls cartilage development by antagonizing Gli3, but requires additional effectors to regulate osteoblast and vascular development. Development 2005; 132: 4339-4351.

16 Joeng KS, Long F. The Gli2 transcriptional activator is a crucial effector for Ihh signaling in osteoblast development and cartilage vascularization. Development 2009; 136: 4177-4185.

17 Vokes SA, Ji H, Wong WH, McMahon AP. A genome-scale analysis of the cis-regulatory circuitry underlying sonic hedgehog-mediated patterning of the mammalian limb. Genes Dev 2008; 22: 2651-2663.

18 Vokes SA, Ji H, McCuine S et al. Genomic characterization of Gli-activator targets in sonic hedgehog-mediated neural patterning. Development 2007; 134: 1977-1989. 
19 Hojo H, Ohba S, Yano F et al. Gli1 protein participates in Hedgehogmediated specification of osteoblast lineage during endochondral ossification. J Biol Chem 2012; 287: 17860-17869.

$20 \mathrm{Tu}$ X, Joeng KS, Long F. Indian hedgehog requires additional effectors besides Runx 2 to induce osteoblast differentiation. Dev Biol 2012; 362: 76-82.

21 Daneman R, Agalliu D, Zhou L et al. Wnt/beta-catenin signaling is required for CNS, but not non-CNS, angiogenesis. Proc Natl Acad Sci USA 2009; 106: 641-646. (c) (i) $(-)$ This work is licensed under a Creative Commons Attribution-

NonCommercial-NoDerivs 3.0 Unported License. The images or other third party material in this article are included in the article's Creative Commons license unless indicated otherwise in the credit line; if the material is not included under the Creative Commons license, users will need to obtain permission from the license holder to reproduce the material. To view a copy of this license, visit http:// creativecommons.org/licenses/by-nc-nd/3.0/ 